\title{
2 Digitalisierung für mehr analogen gesundheitlichen Wohlstand
}

\author{
Astrid Böger
}

\section{Bestandsaufnahme und Stellenwert}

Die Herausforderungen der Digitalisierung bestimmen gegenwärtig die Diskussionen im Gesundheitswesen. Kongresse, Publikationen, Workshops widmen sich diesen Themen. Dabei stehen vor allem die technologischen Möglichkeiten durch den Einsatz von Plattformen, Portalen sowie Fragen zur Interoperabilität, zu Standards oder die Sicherheit der Vernetzung und Datenschutzthemen im Vordergrund. Es geht darum, Zeit zu sparen, Kosten zu reduzieren, Prozesse zu verschlanken.

Verbesserungen der Behandlungsqualität und höhere Lebensqualität der Patienten rücken dabei in den Vordergrund. Das steht bisher in unserem Gesundheitssystem unter wirtschaftlichen Zwängen keineswegs im Mittelpunkt, so paradox das klingt.

Oftmals scheinen die Konsequenzen des Einsatzes der Digitalisierung naheliegend, obwohl die Komplexität der Veränderungen, die sich nicht nur im Rahmen der Versorgungsprozesse sondern auf der Werte- und Normenebene abspielen, viel weitreichender zu sein. Drei Szenarien bestimmen die Diskussion:

Die Digitalisierung löst die bisherigen analogen Prozesse weitestgehend ab und verdrängt konsequent analoge Handlungsoptionen aus der Praxis. Die Digitalisierung positioniert sich gleichberechtigt zu den gegenwärtigen analogen Prozessen oder aber es kommt zu einem „mehr“ an menschlichen Entscheidungs- und Handlungsoptionen.

Diese letzte Option erscheint auf den ersten Blick für die Diskussion wiedersinnig. Wir sprechen flächendeckend von der Notwendigkeit, stärker auf die Digitalisierung 
im Gesundheitswesen zu fokussieren und zielen eigentlich auf das Erstarken der Arzt-Patient Beziehungen? Aber das ist der nachvollziehbar vernünftige Ansatz und muss entlang einer plausiblen Argumentationskette zukünftig stärker in den Vordergrund gerückt werden.

Wo liegen hier die Herausforderungen?

\section{Charakteristika des Transformationsprozesses}

Grundansatz für diese Annahme bilden die Prinzipien des „invisible Computings“ oder auch der „embedded technologies“ und des IoTs (Internet of Things) sowie die Robotik für Diagnostik und OP.

Gegenwärtige Versorgungsmodelle werden durch diese Art des Einsatzes neuer Technologien aufgebrochen und verändern Kalkulationen und Herangehensweisen im Gesundheitswesen. Wissens-, Beratungs- aber auch Behandlungsprozesse verlassen die traditionellen Wege, die sich vor allem durch einen direkten Arzt-Patient-Kontakt ausgezeichnet haben. In diesen Direktkontakt drängen nun Technologien, die zu einem veränderten Verhältnis der Akteure führen. Gleichzeitig führt dies zu einer Verschiebung der Machtkonstellation um das Geschäftsfeld „Gesundheit“.

Befindet sich die „Digitalisierungsmacht“ vor allem bei den Akteuren (Ärzten, Krankenkassen, Versicherungen, Medizinischen Einrichtungen) ist davon auszugehen, dass die Daten vor allem dazu verwendet werden, zu rationalisieren und diese im Sinne einer Geschäftsoptimierung zu nutzen. Das schließt den Ausbau von gesundheitsbeeinflussenden Manipulationsszenarien ein.

Liegt die „Digitalisierungsmacht“ bei den Patienten kann es perspektivisch zu einer „Aufklärung“ des Menschen über seine gesundheitsbeeinflussenden Faktoren in einem über die letzten Jahrzehnte bis zur Perfektion ausgebauten „medizinischen Businessplanspiel“ kommen und damit auch zeitnah zu einem volkswirtschaftlich wirksameren Cesundheitssystem.

Dabei bestehen durch die neuen Technologien erstmals die Chancen, proaktiv den Fokus auf den Erhalt der Gesundheit zu konzentrieren und somit den Wettlauf negativer Veränderungsprozesse im Sinne menschlicher Erkrankungen zu gewinnen.

Dazu gehören Software zur Erkennung und Verhinderung der Verbreitung von Keimen, zur Verbesserung von Hygieneprozessen in Krankenhäusern, Filtersysteme zur Verhinderung von krankmachenden Verschmutzungen, Textilien, die krankmachende Strahlen abschirmen, mobile Messstationen, die schädliche Luftkonzentrationen messen und rechtzeitig davor warnen, Technologien, die es ermöglichen, dass Menschen nur noch halbtags arbeiten und damit Stress entfällt, Erkenntnisse, dass der Ressourcenverbrauch und damit die Produktion halbiert werden können, Wissen darüber, dass der Mensch weniger Nahrung benötigt, weniger Fleisch, weniger Fertigprodukte, dadurch weniger krank wird, weniger pharmazeutische Unterstützung benötigt, weniger medizinische Versorgungsprozesse in Anspruch nehmen muss, damit im Gesundheitssystem weniger Kosten verursacht werden.

Allerdings bedeutet dies im Umkehrschluss nicht, das prädiktive und personalisierte Mess- und Analyseverfahren per se als negativ zu betrachten sind, sondern dass natürlich auch im Zusammenhang mit genetischen Tests, der Erschließung neuro- 
naler Prozesse oder dem langfristigen Monitoring unterschiedlicher Körperfunktionen vorbeugend Krankheitsrisiken begegnet werden kann.

Das Ziel der Digitalisierung im Gesundheitswesen sollte auf eine, mittels Digitalisierung unterstützte, dringend notwendige Aufklärungs-, Transparenz- und Bildungsoffensive abzielen, um mit dieser die Grundlagen für eine verbesserte Gesundheit der einzelnen Bürger zu legen.

Maßnahmen der Digitalisierung müssen neben dem Erkenntnisgewinn für die Medizin vor allem zu einer Erhöhung des persönlichen Wohlfühlens führen, einschließlich der Erhöhung des persönlichen Zeitwohlstands, der Zeitsouveränität und der Qualitätszeit, anstatt Stress im Arbeits- und Freizeitbereich zu generieren.

In der heutigen Zeit liegen fast alle Erkenntnisse vor, die zu Erkrankungen von Menschen führen:

- Schädigende Emissionen (z.B. Lärm, Licht, Schmutz, Strahlen),

- Stress, Hektik,

- Keime, Viren, Bakterien,

- Genussmittel, Produkte der Ernährung, Gifte (Pharma) und

- Süchte, Phobien, psychische Experimente, emotionale Steuerungen (u.a. mediale Manipulationen), um einmal die wichtigsten Gruppen zu nennen.

So, wie die Erkenntnisse zu den Erkrankungen vorliegen, sind gleichermaßen die Umfeldindikatoren und Beeinflussungsfaktoren bekannt, deren Veränderungen zu einer Eindämmung von Erkrankungen führen können.

Um dies aber flächendeckend zu erreichen, sind Aufklärungskampagnen notwendig, die genau dieses Wissen transportieren und weitergeben. Hierfür muss digitales Wissen genutzt werden, wie einst bei Einführung des Buchdrucks analoges.

Vor gleichen revolutionären Umbrüchen stehen wir nun mit der massiven Einführung von Digitalisierungsprozessen, die die Machtkonstellationen sowohl in die eine als auch die andere Richtung verschieben können.

\section{Chancen und Risiken}

Während die Sammlung und Verknüpfung von Daten vielfach als positiver Beitrag zur Verbesserung der Versorgungsforschung gesehen wird, besteht ein großes Risiko, dass personalisierte Prozesse zu einem massiven Missbrauch der Datensammlungen führen.

Andererseits besteht die Chance, all die in den letzten Jahrzehnten gewonnenen Erkenntnisse so transparent zu machen, dass es zu einem positiven Umsteuern innerhalb des Gesundheitswesens kommen kann.

In den Debatten, Diskussionen aber auch bei der Initiierung von Projekten sollte vor allem auf die Ausrichtung der Konzepte Wert gelegt werden. Hier muss es im Vorfeld der Einführung zu einer umfassenden Bewertung durch die Gesellschaft kommen, welche Digitalisierungsprojekte langfristig positive Folgen für den Menschen, den Patienten und die Akteure haben und welche genau das Gegenteil bewirken.

Ein Beispiel für eine Digitalisierung stellt die komplexe Einführung von Entertainmentsystemen in Krankenhäusern dar. Während viele von einer positiven Wirkung für den Aufenthalt des Patienten ausgehen, trägt solch ein System aber eher weniger 
dazu bei, den Gesundungsprozess zu befördern, der vor allem durch Schlaf und Ruhe bewirkt wird und nicht durch zusätzliche Beeinflussungen und mentale Stressoren.

Auch der umfassende Einsatz von Telemonitoring-Lösungen trägt nur in geringem Maße zu einer volkswirtschaftlichen Verbesserung im Gesundheitswesen bei. Vielmehr dienen diese Projekte dazu, die Big-Data-Konten der Betreiber zu füllen und das so gewonnene Datengold in andere Geschäftsmodelle rund um die Krankheit der Patienten einfließen zu lassen.

Nicht umsonst finden Projekte zu Telediagnostik, Teletherapie oder Telekonsultationen eher weniger Eingang in das Gesundheitssystem, da diese keine Daten erzeugen, sondern vielmehr den Patienten befähigen, schneller wieder das Gesundheitssystem zu verlassen.

Da gilt es auch an jahrhundertealte bewährte einfache Erkenntnisse zu erinnern:

Würde es zum Allgemeinwissen der Menschen gehören, dass mit Heilfasten zahlreiche Erkrankungen wirkungsvoll bekämpft und eingedämmt werden können, würden ganzen Ärztenetzwerken, Krankenhausbetreibern, Pharmaunternehmen ihre Geschäftsgrundlagen entzogen.

Aber in diesem Sinne geht es bei der Digitalisierung auch darum, altbewährtes Gesundheitswissen nicht zu vergessen, sondern über eine Demokratisierung, Liberalisierung und Transparenz hinsichtlich gesundheitlicher Prozesse die Herstellung einer „medizinischen Literacy“ in der Breite der Bevölkerung zu befördern.

\section{Das oberste Digitalisierungsziel sollte es demnach sein, dem Menschen sichtbar zu machen, welche Indikatoren für den Komplex der persön- lichen Gesundheit verantwortlich sind und ihn zu befähigen, diese In- dikatoren positiv und eigenverantwortlich zu verändern.}

\section{Trends und Entwicklungen}

Eingesetzte Technologien dafür finden sich z.B. im Bereich der Biofeedback- und Neurofeedbacktechnologien, aber auch der Wearables, die durch die persönliche „Selbstvermessung“ dazu beitragen, die Achtsamkeit für und die Erkenntnisse über die eigenen Körperfunktionen in Abhängigkeit von schmerzhaften und nicht schmerzenden, kranken und gesunden Zuständen sowie deren Auslösern zu erhöhen.

Der Gewinn aus der Digitalisierung besteht dann in der Zunahme positiver ,analoger" Prozesse im Sinne von Zeit- und gesundheitlichem Wohlstand. Die digitale Beschleunigung geht somit mit einer analogen Entschleunigung im realen Leben einher und führt, auch in allen anderen gesellschaftlichen Bereichen, adäquat zu mehr Wohlstand - ökologischem Wohlstand für die Gesellschaft, sozialem und kulturellem Wohlstand und eben dem gesundheitlichen Wohlstand des Einzelnen. 